Article

\title{
Quantum Integral Inequalities of Simpson-Type for Strongly Preinvex Functions
}

\author{
Yongping Deng ${ }^{1}$, Muhammad Uzair Awan ${ }^{2}$ (D) and Shanhe $\mathrm{Wu}^{1, *(\mathbb{D})}$ \\ 1 Department of Mathematics, Longyan University, Longyan 364012, China \\ 2 Department of Mathematics, Government College University, Faisalabad 38000, Pakistan \\ * Correspondence: shanhewu@gmail.com
}

Received: 29 June 2019; Accepted: 13 August 2019; Published: 16 August 2019

check for updates

\begin{abstract}
In this paper, we establish a new $q$-integral identity, the result is then used to derive two $q$-integral inequalities of Simpson-type involving strongly preinvex functions. Some special cases of the obtained results are also considered, it is shown that several new and previously known results can be derived via generalized strongly preinvex functions and quantum integrals.
\end{abstract}

Keywords: $q$-integral inequality; strongly preinvex function; Simpson inequality; quantum integral

MSC: 26D10; 26D15; 26A51; 34A08

\section{Introduction}

Quantum calculus or q-calculus is often known as "calculus without limits" and was first developed by Jackson in the early twentieth century, but the history of quantum calculus can be traced back to some much earlier work done by Euler and Jacobi et al. (see [1]). Over the recent decade, the investigation of $q$-calculus has attracted the interest of many researchers, because it has been found to have a lot of applications in mathematics and physics. As is known to us, $q$-calculus can be treated as a bridge between mathematics and physics, it is a significant tool for researchers working in analytic number theory, noncommutative geometry, or theoretical physics. In quantum calculus, we obtain the $q$-analogues of mathematical objects which can be recaptured as $q \rightarrow 1^{-}$. It has been noticed that quantum calculus is a subfield of timescale calculus. Timescale calculus provides a unified framework for studying dynamic equations on both the discrete and continuous domains. In quantum calculus, we are concerned with a specific timescale, called the $q$-timescale (see [1-4]).

The concept of convexity has been extended in several directions, since these generalized versions have significant applications in different fields of pure and applied sciences. We only point out that convexity was recently used in differential geometry to completely classify ideal Casorati submanifolds in complex space forms (see [5-8]). One of the convincing examples on extensions of convexity is the introduction of invex function, which was introduced by Hanson [9]. This concept is particularly interesting from an optimization viewpoint, since it provides a broader setting to study the optimization and mathematical programming problems. Such optimization problems have recently been considered in Riemannian geometry by an original choice of a set of quadratic programming problems. Since then, some classes of generalized convex functions, such as the preinvex function, strongly $\alpha$-invex function, and strongly $\alpha$-preinvex function, were put forward successively, see [10-16].

In this paper, the quantum calculus and the strongly preinvex function are subtly linked together via integral inequalities. It is well known that the theory of inequality plays a fundamental role in pure and applied mathematics and has extensive applications. Apart from the larger number of research results of inequalities in classical analysis, there are considerable works on the study of inequalities for $q$-calculus, particularly the study of inequalities related to quantum integral ( $q$-integral), for 
example, $q$-Hermite-Hadamard integral inequality, $q$-Cauchy-Schwarz integral inequality, $q$-Hölder integral inequality, $q$-Ostrowski integral inequality, etc. For more details, we refer the interested reader to [17-23] and the references cited therein.

The purpose of this paper is to establish several $q$-integral inequalities of Simpson-type via strongly preinvex functions. The classical Simpson inequality is described as follows:

$$
\left|\frac{1}{6}\left[\phi(\alpha)+4 \phi\left(\frac{\alpha+\beta}{2}\right)+\phi(\beta)\right]-\frac{1}{\beta-\alpha} \int_{\alpha}^{\beta} \phi(v) d v\right| \leq \frac{1}{1280}\left\|\phi^{(4)}\right\|_{\infty}(\beta-\alpha)^{4},
$$

where the mapping $\phi:[\alpha, \beta] \rightarrow R$ is four times continuously differentiable, and $\left\|\phi^{(4)}\right\|_{\infty}=\sup _{v \in(\alpha, \beta)}\left|\phi^{(4)}(v)\right|<\infty$ (see [24]).

The paper is organized as follows: In Sections 2 and 3, we shall introduce some notions and properties on strongly preinvex functions and $q$-calculus. As an auxiliary result, we present an identity associated with $q$-integral. In Section 4 , with the help of the auxiliary result, we will establish our main results. At the end of the paper, some examples are provided to illustrate the applications of our main results.

\section{Preliminaries}

Let us recall some preliminary concepts and results.

Definition 1 ([15]). A set $K_{\eta} \subset \mathbb{R}^{n}$ is said to be invex with respect to bifunction $\eta(.,):. \mathbb{R}^{n} \times \mathbb{R}^{n} \rightarrow \mathbb{R}^{n}$, if

$$
u+\lambda \eta(v, u) \in K_{\eta}, \quad \forall u, v \in K_{\eta}, \lambda \in[0,1] .
$$

Definition 2 ([15]). A function $\phi$ on the invex set $K_{\eta} \subset \mathbb{R}^{n}$ is said to be preinvex with respect to bifunction $\eta(.,):. \mathbb{R}^{n} \times \mathbb{R}^{n} \rightarrow \mathbb{R}^{n}$, if

$$
\phi(u+\lambda \eta(v, u)) \leq(1-\lambda) \phi(u)+\lambda \phi(v), \quad \forall u, v \in K_{\eta}, \lambda \in[0,1] .
$$

Definition 3 ([16]). A function $\phi$ on the invex set $K_{\eta} \subset \mathbb{R}^{n}$ is said to be strongly preinvex with respect to bifunction $\eta(.,):. \mathbb{R}^{n} \times \mathbb{R}^{n} \rightarrow \mathbb{R}^{n}$, and modulus $\mu>0$, if

$$
\phi(u+\lambda \eta(v, u)) \leq(1-\lambda) \phi(u)+\lambda \phi(v)-\mu \lambda(1-\lambda) \eta^{2}(v, u), \quad \forall u, v \in K_{\eta}, \lambda \in[0,1] .
$$

Here, we introduce a new definition which combines the preinvex functions and the strongly preinvex functions given above.

Definition 4. A function $\phi$ on the invex set $K_{\eta} \subset \mathbb{R}^{n}$ is said to be generalized strongly preinvex with respect to bifunction $\eta(.,):. \mathbb{R}^{n} \times \mathbb{R}^{n} \rightarrow \mathbb{R}^{n}$ and modulus $\mu \geq 0$, if

$$
\phi(u+\lambda \eta(v, u)) \leq(1-\lambda) \phi(u)+\lambda \phi(v)-\mu \lambda(1-\lambda) \eta^{2}(v, u), \quad \forall u, v \in K_{\eta}, \lambda \in[0,1] .
$$

Clearly, if $\mu=0$, then the class of generalized strongly preinvex functions reduces to the class of preinvex functions as defined in Definition 2.

In the following, we recall some basic properties of $q$-calculus.

Let $J=[a, b] \subseteq \mathbb{R}$ be an interval and $0<q<1$ be a constant. The $q$-derivative of a function $\phi: J \rightarrow \mathbb{R}$ at a point $u \in J$ on $[a, b]$ is defined as follows:

Definition 5 ([25]). Let $\phi: J=[a, b] \rightarrow \mathbb{R}$ be a continuous function and let $u \in J$. Then, the $q$-derivative of $\phi$ on $J$ at $u$ is defined as 


$$
{ }_{a} D_{q} \phi(u)=\frac{\phi(u)-\phi(q u+(1-q) a)}{(1-q)(u-a)}, \quad u \neq a .
$$

Definition 6 ([25]). Let $\phi: J=[a, b] \rightarrow \mathbb{R}$ is a continuous function. A second-order $q$-derivative on $J$, which is denoted as ${ }_{a} D_{q}^{2} \phi$, provided that ${ }_{a} D_{q} \phi$ is $q$-differentiable on $J$ with ${ }_{a} D_{q}^{2} \phi={ }_{a} D_{q}\left({ }_{a} D_{q} \phi\right): J \rightarrow \mathbb{R}$. Similarly higher order $q$-derivative on $J$ is defined by ${ }_{a} D_{q}^{n} \phi: J \rightarrow \mathbb{R}$.

In [25], Tariboon and Ntouyas defined the $q$-integral as follows:

Definition 7 ([25]). Let $\phi: J=[a, b] \rightarrow \mathbb{R}$ be a continuous function. Then, the q-integral on $J$ is defined as:

$$
\int_{a}^{u} \phi(v){ }_{a} \mathrm{~d}_{q} v=(1-q)(u-a) \sum_{n=0}^{\infty} q^{n} \phi\left(q^{n} u+\left(1-q^{n}\right) a\right),
$$

for $u \in J$.

The following results are useful in the computation of $q$-integral in subsequent section.

Proposition 1 ([25]). Let $f, g: J=[a, b] \rightarrow \mathbb{R}$ be continuous functions, $c \in \mathbb{R}$. Then, for $x \in J$,

$$
\begin{aligned}
& \int_{a}^{x}(f(v)+g(v))_{a} \mathrm{~d}_{q} v=\int_{a}^{x} f(v)_{a} \mathrm{~d}_{q} v+\int_{a}^{x} f(v)_{a} \mathrm{~d}_{q} v, \\
& \int_{a}^{x} c f(v)_{a} \mathrm{~d}_{q} v=c \int_{a}^{x} f(v)_{a} \mathrm{~d}_{q} v, \\
& \int_{\xi}^{x} f(v)_{a} \mathrm{~d}_{q} v=\int_{a}^{x} f(v)_{a} \mathrm{~d}_{q} v-\int_{a}^{\xi} f(v){ }_{a} \mathrm{~d}_{q} v, \quad \xi \in(a, x) .
\end{aligned}
$$

Proposition 2 ([25]). For q-integral, we have the following identities

$$
\begin{aligned}
\int_{a}^{u} 1_{a} \mathrm{~d}_{q} v & =u-a, \\
\int_{a}^{u} v{ }_{a} \mathrm{~d}_{q} v & =\frac{(u-a)(u+q a)}{1+q}, \\
\int_{a}^{u}(v-a)^{\tau}{ }_{a} \mathrm{~d}_{q} v & =\left(\frac{1-q}{1-q^{\tau+1}}\right)(u-a)^{\tau+1}, \quad \tau \neq-1, \\
\int_{\xi}^{u}(v-\xi){ }_{a} \mathrm{~d}_{q} v & =\frac{u^{2}-(1+q) u \xi+q \xi^{2}}{1+q}-\frac{a(1-q)(u-\xi)}{1+q}, \quad \xi \in(a, u) .
\end{aligned}
$$

\section{A Key Lemma}

In this section, we present an identity associated with $q$-integral, which plays an important role in establishing our main results.

Lemma 1. Let $f: I=[a, a+\eta(b, a)] \rightarrow \mathbb{R}$ be a $q$-differentiable function on I with $\eta(b, a)>0$. If ${ }_{a} \mathrm{D}_{q} f$ is integrable on I and $0<q<1$, then

$$
\begin{aligned}
& \frac{1}{6}\left[f(a)+4 f\left(\frac{2 a+\eta(b, a)}{2}\right)+f(a+\eta(b, a))\right]-\frac{1}{\eta(b, a)} \int_{a}^{a+\eta(b, a)} f(t)_{a} \mathrm{~d}_{q} t \\
& =\eta(b, a) \int_{0}^{1} \Psi(t, q)_{a} \mathrm{D}_{q} f(a+t \eta(b, a))_{0} \mathrm{~d}_{q} t
\end{aligned}
$$


where

$$
\Psi(t, q)= \begin{cases}q t-\frac{1}{6}, & \text { if } 0 \leq t<\frac{1}{2} \\ q t-\frac{5}{6}, & \text { if } \frac{1}{2} \leq t \leq 1\end{cases}
$$

Proof. Let

$$
\begin{aligned}
& Q_{1}=\int_{0}^{\frac{1}{2}}\left(q t-\frac{1}{6}\right){ }_{a} \mathrm{D}_{q} f(a+t \eta(b, a))_{0} \mathrm{~d}_{q} t \\
& Q_{2}=\int_{\frac{1}{2}}^{1}\left(q t-\frac{5}{6}\right){ }_{a} \mathrm{D}_{q} f(a+t \eta(b, a))_{0} \mathrm{~d}_{q} t
\end{aligned}
$$

then

$$
\int_{0}^{1} \Psi(t, q){ }_{a} \mathrm{D}_{q} f(a+t \eta(b, a)){ }_{0} \mathrm{~d}_{q} t=Q_{1}+Q_{2} .
$$

Utilizing the Definitions 5 and 7 , and the properties of $q$-derivative and $q$-integral described in Propositions 1, a direct computation gives

$$
\begin{aligned}
& Q_{1}=\int_{0}^{\frac{1}{2}} q t_{a} \mathrm{D}_{q} f(a+t \eta(b, a)){ }_{0} \mathrm{~d}_{q} t-\int_{0}^{\frac{1}{2}} \frac{1}{6}{ }_{a} \mathrm{D}_{q} f(a+t \eta(b, a))_{0} \mathrm{~d}_{q} t \\
& =\int_{0}^{\frac{1}{2}} q \frac{f(a+t \eta(b, a))-f(a+q t \eta(b, a))}{(1-q) \eta(b, a)} 0 \mathrm{~d}_{q} t \\
& -\frac{1}{6} \int_{0}^{\frac{1}{2}} \frac{f(a+t \eta(b, a))-f(a+q t \eta(b, a))}{(1-q) \operatorname{t\eta }(b, a)}{ }_{0} \mathrm{~d}_{q} t \\
& =\frac{1}{2} \sum_{n=0}^{\infty} q^{n+1} \frac{f\left(\frac{2 a+q^{n} \eta(b, a)}{2}\right)-f\left(\frac{2 a+q^{n+1} \eta(b, a)}{2}\right)}{\eta(b, a)} \\
& -\frac{1}{6} \sum_{n=0}^{\infty} \frac{f\left(\frac{2 a+q^{n} \eta(b, a)}{2}\right)-f\left(\frac{2 a+q^{n+1} \eta(b, a)}{2}\right)}{\eta(b, a)} \\
& =\frac{1}{2}\left[\frac{f\left(\frac{2 a+\eta(b, a)}{2}\right)}{\eta(b, a)}-\sum_{n=0}^{\infty}(1-q) q^{n} \frac{f\left(\frac{2 a+q^{n} \eta(b, a)}{2}\right)}{\eta(b, a)}\right]-\frac{1}{6} \cdot \frac{f\left(\frac{2 a+\eta(b, a)}{2}\right)-f(a)}{\eta(b, a)} \\
& =\frac{1}{3} \cdot \frac{f\left(\frac{2 a+\eta(b, a)}{2}\right)}{\eta(b, a)}+\frac{1}{6} \cdot \frac{f(a)}{\eta(b, a)}-\frac{1}{2} \sum_{n=0}^{\infty}(1-q) q^{n} \frac{f\left(\frac{2 a+q^{n} \eta(b, a)}{2}\right)}{\eta(b, a)} \\
& =\frac{1}{3} \cdot \frac{f\left(\frac{2 a+\eta(b, a)}{2}\right)}{\eta(b, a)}+\frac{1}{6} \cdot \frac{f(a)}{\eta(b, a)}-\frac{1}{\eta(b, a)} \int_{0}^{\frac{1}{2}} f(a+t \eta(b, a))_{0} \mathrm{~d}_{q} t .
\end{aligned}
$$

On the other hand, one has

$$
\begin{gathered}
\left.Q_{2}=\int_{\frac{1}{2}}^{1} q t_{a} \mathrm{D}_{q} f(a+t \eta(b, a))\right)_{0} \mathrm{~d}_{q} t-\int_{\frac{1}{2}}^{1} \frac{5}{6}{ }_{a} \mathrm{D}_{q} f(a+t \eta(b, a))_{0} \mathrm{~d}_{q} t \\
=\int_{0}^{1} q t_{a} \mathrm{D}_{q} f(a+t \eta(b, a))_{0} \mathrm{~d}_{q} t-\int_{0}^{1} \frac{5}{6}{ }_{a} \mathrm{D}_{q} f(a+t \eta(b, a))_{0} \mathrm{~d}_{q} t \\
-\left(\int_{0}^{\frac{1}{2}} q t_{a} \mathrm{D}_{q} f(a+t \eta(b, a))_{0} \mathrm{~d}_{q} t-\int_{0}^{\frac{1}{2}} \frac{5}{6}{ }_{a} \mathrm{D}_{q} f(a+t \eta(b, a))_{0} \mathrm{~d}_{q} t\right) .
\end{gathered}
$$

Since 


$$
\begin{gathered}
\int_{0}^{1} q t_{a} \mathrm{D}_{q} f(a+t \eta(b, a))_{0} \mathrm{~d}_{q} t-\int_{0}^{1} \frac{5}{6}{ }_{a} \mathrm{D}_{q} f(a+t \eta(b, a))_{0} \mathrm{~d}_{q} t \\
=\int_{0}^{1} q \frac{f(a+t \eta(b, a))-f(a+q t \eta(b, a))}{(1-q) \eta(b, a)}{ }_{0} \mathrm{~d}_{q} t \\
\quad-\frac{5}{6} \int_{0}^{1} \frac{f(a+t \eta(b, a))-f(a+q t \eta(b, a))}{(1-q) t \eta(b, a)}{ }_{0} \mathrm{~d}_{q} t \\
=\sum_{n=0}^{\infty} q^{n+1} \frac{f\left(a+q^{n} \eta(b, a)\right)-f\left(a+q^{n+1} \eta(b, a)\right)}{\eta(b, a)} \\
\quad-\frac{5}{6} \sum_{n=0}^{\infty} \frac{f\left(a+q^{n} \eta(b, a)\right)-f\left(a+q^{n+1} \eta(b, a)\right)}{\eta(b, a)} \\
=\frac{f(a+\eta(b, a))}{\eta(b, a)}-\sum_{n=0}^{\infty}(1-q) q^{n} \frac{f\left(a+q^{n} \eta(b, a)\right)}{\eta(b, a)}-\frac{5}{6} \cdot \frac{f(a+\eta(b, a))-f(a)}{\eta(b, a)} \\
=\frac{1}{6} \cdot \frac{f(a+\eta(b, a))}{\eta(b, a)}+\frac{5}{6} \cdot \frac{f(a)}{\eta(b, a)}-\sum_{n=0}^{\infty}(1-q) q^{n} \frac{f\left(a+q^{n} \eta(b, a)\right)}{\eta(b, a)} \\
=\frac{1}{6} \cdot \frac{f(a+\eta(b, a))}{\eta(b, a)}+\frac{5}{6} \cdot \frac{f(a)}{\eta(b, a)}-\frac{1}{\eta(b, a)} \int_{0}^{1} f(a+t \eta(b, a)){ }_{0} \mathrm{~d}_{q} t
\end{gathered}
$$

and

$$
\begin{gathered}
\int_{0}^{\frac{1}{2}} q t_{a} \mathrm{D}_{q} f(a+t \eta(b, a))_{0} \mathrm{~d}_{q} t-\int_{0}^{\frac{1}{2}} \frac{5}{6}{ }_{a} \mathrm{D}_{q} f(a+t \eta(b, a))_{0} \mathrm{~d}_{q} t \\
=\frac{1}{2}\left[\frac{f\left(\frac{2 a+\eta(b, a)}{2}\right)}{\eta(b, a)}-\sum_{n=0}^{\infty}(1-q) q^{n} \frac{f\left(\frac{2 a+q^{n} \eta(b, a)}{2}\right)}{\eta(b, a)}\right]-\frac{5}{6} \cdot \frac{f\left(\frac{2 a+\eta(b, a)}{2}\right)-f(a)}{\eta(b, a)} \\
=-\frac{1}{3} \cdot \frac{f\left(\frac{2 a+\eta(b, a)}{2}\right)}{\eta(b, a)}+\frac{5}{6} \cdot \frac{f(a)}{\eta(b, a)}-\frac{1}{\eta(b, a)} \int_{0}^{\frac{1}{2}} f(a+t \eta(b, a))_{0} \mathrm{~d}_{q} t,
\end{gathered}
$$

we obtain

$$
\begin{gathered}
Q_{2}=\frac{1}{6} \cdot \frac{f(a+\eta(b, a))}{\eta(b, a)}+\frac{1}{3} \cdot \frac{f\left(\frac{2 a+\eta(b, a)}{2}\right)}{\eta(b, a)}-\frac{1}{\eta(b, a)} \int_{0}^{1} f(a+t \eta(b, a))_{0} \mathrm{~d}_{q} t \\
+\frac{1}{\eta(b, a)} \int_{0}^{\frac{1}{2}} f(a+t \eta(b, a))_{0} \mathrm{~d}_{q} t
\end{gathered}
$$

Thus,

$$
\begin{gathered}
\int_{0}^{1} \Psi(t, q){ }_{a} \mathrm{D}_{q} f(a+t \eta(b, a))_{0} \mathrm{~d}_{q} t=Q_{1}+Q_{2} \\
=\frac{1}{6} \cdot \frac{f(a+\eta(b, a))+f(a)}{\eta(b, a)}+\frac{2}{3} \cdot \frac{f\left(\frac{2 a+\eta(b, a)}{2}\right)}{\eta(b, a)}-\frac{1}{\eta(b, a)} \int_{0}^{1} f(a+t \eta(b, a))_{0} \mathrm{~d}_{q} t \\
=\frac{1}{6} \cdot \frac{f(a+\eta(b, a))+f(a)}{\eta(b, a)}+\frac{2}{3} \cdot \frac{f\left(\frac{2 a+\eta(b, a)}{2}\right)}{\eta(b, a)}-\frac{1}{\eta^{2}(b, a)} \int_{a}^{a+\eta(b, a)} f(t)_{a} \mathrm{~d}_{q} t,
\end{gathered}
$$

which leads to the desired identity (4). The proof of Lemma 1 is complete. 


\section{Main Results}

We are in a position to establish the $q$-integral inequalities of Simpson-type for strongly preinvex functions.

Theorem 1. Let $f: I=[a, a+\eta(b, a)] \rightarrow \mathbb{R}$ be a q-differentiable function on I with $\eta(b, a)>0$. If $\left|{ }_{a} \mathrm{D}_{q} f\right|$ is an integrable and a generalized strongly preinvex function with modulus $\mu \geq 0$ and $0<q<1$, then

$$
\begin{aligned}
& \left|\frac{1}{6}\left[f(a)+4 f\left(\frac{2 a+\eta(b, a)}{2}\right)+f(a+\eta(b, a))\right]-\frac{1}{\eta(b, a)} \int_{a}^{a+\eta(b, a)} f(t) a_{a} \mathrm{~d}_{q} t\right| \\
& \leq \eta(b, a)\left[\left(A_{1}(q)+A_{4}(q)\right)\left|{ }_{a} \mathrm{D}_{q} f(a)\right|+\left(A_{2}(q)+A_{5}(q)\right)\left|{ }_{a} \mathrm{D}_{q} f(b)\right|-\mu\left(A_{3}(q)+A_{6}(q)\right) \eta^{2}(b, a)\right],
\end{aligned}
$$

where $A_{1}(q), A_{2}(q), A_{3}(q), A_{4}(q), A_{5}(q)$, and $A_{6}(q)$ are given by

$$
\begin{aligned}
& A_{1}(q)=\left\{\begin{array}{l}
\frac{1-4 q^{3}}{24(1+q)\left(1+q+q^{2}\right)}, \quad 0<q<\frac{1}{3} \\
\frac{1+12 q+12 q^{2}+36 q^{3}}{216(1+q)\left(1+q+q^{2}\right)}, \frac{1}{3} \leq q<1
\end{array}\right. \\
& A_{2}(q)=\left\{\begin{array}{l}
\frac{1-2 q-2 q^{2}}{24(1+q)\left(1+q+q^{2}\right)}, \quad 0<q<\frac{1}{3} \\
\frac{18 q^{2}+18 q-7}{216(1+q)\left(1+q+q^{2}\right)}, \frac{1}{3} \leq q<1
\end{array}\right. \\
& A_{3}(q)= \begin{cases}\frac{1-2 q-2 q^{3}-4 q^{4}}{48(1+q)\left(1+q^{2}\right)\left(1+q+q^{2}\right)}, & 0<q<\frac{1}{3} \\
\frac{108 q^{4}+54 q^{3}+12 q^{2}+54 q-17}{1296(1+q)\left(1+q^{2}\right)\left(1+q+q^{2}\right)}, & \frac{1}{3} \leq q<1\end{cases} \\
& A_{4}(q)=\left\{\begin{array}{l}
\frac{-5+8 q+8 q^{2}-8 q^{3}}{24(1+q)\left(1+q+q^{2}\right)}, \quad 0<q<\frac{5}{6} \\
\frac{12 q^{2}+12 q+5}{216(1+q)\left(1+q+q^{2}\right)}, \frac{5}{6} \leq q<1
\end{array}\right. \\
& A_{5}(q)= \begin{cases}\frac{5-2 q-2 q^{2}}{8(1+q)\left(1+q+q^{2}\right)}, & 0<q<\frac{5}{6} \\
\frac{18 q^{2}+18 q+25}{216(1+q)\left(1+q+q^{2}\right)}, & \frac{5}{6} \leq q<1\end{cases} \\
& A_{6}(q)= \begin{cases}\frac{5-2 q+28 q^{2}-2 q^{3}-12 q^{4}}{48(1+q)\left(1+q^{2}\right)\left(1+q+q^{2}\right)}, & 0<q<\frac{5}{6} \\
\frac{108 q^{4}-54 q^{3}+96 q^{2}-54 q+115}{1296(1+q)\left(1+q^{2}\right)\left(1+q+q^{2}\right)}, & \frac{5}{6} \leq q<1 .\end{cases}
\end{aligned}
$$

Proof. Using Lemma 1 and the assumption condition that $\left|{ }_{a} \mathrm{D}_{q} f\right|$ is a generalized strongly preinvex function, we have

$$
\left|\frac{1}{6}\left[f(a)+4 f\left(\frac{2 a+\eta(b, a)}{2}\right)+f(a+\eta(b, a))\right]-\frac{1}{\eta(b, a)} \int_{a}^{a+\eta(b, a)} f(t){ }_{a} \mathrm{~d}_{q} t\right|
$$




$$
\begin{aligned}
& =\left|\eta(b, a) \int_{0}^{1} \Psi(t, q){ }_{a} \mathrm{D}_{q} f(a+t \eta(b, a)){ }_{0} \mathrm{~d}_{q} t\right| \\
& =\eta(b, a) \mid \int_{0}^{\frac{1}{2}}\left(q t-\frac{1}{6}\right){ }_{a} \mathrm{D}_{q} f(a+t \eta(b, a))_{0} \mathrm{~d}_{q} t \\
& +\int_{\frac{1}{2}}^{1}\left(q t-\frac{5}{6}\right){ }_{a} \mathrm{D}_{q} f(a+t \eta(b, a))_{0} \mathrm{~d}_{q} t \\
& \leq \eta(b, a)\left[\int_{0}^{\frac{1}{2}}\left|q t-\frac{1}{6}\right|\left|{ }_{a} \mathrm{D}_{q} f(a+t \eta(b, a))\right|{ }_{0} \mathrm{~d}_{q} t\right. \\
& \left.+\int_{\frac{1}{2}}^{1}\left|q t-\frac{5}{6}\right|\left|{ }_{a} \mathrm{D}_{q} f(a+t \eta(b, a))\right|{ }_{0} \mathrm{~d}_{q} t\right] \\
& \leq \eta(b, a)\left[\int_{0}^{\frac{1}{2}}\left|q t-\frac{1}{6}\right|\left((1-t)\left|{ }_{a} \mathrm{D}_{q} f(a)\right|+t\left|{ }_{a} \mathrm{D}_{q} f(b)\right|-\mu t(1-t) \eta^{2}(b, a)\right){ }_{0} \mathrm{~d}_{q} t\right. \\
& \left.+\int_{\frac{1}{2}}^{1}\left|q t-\frac{5}{6}\right|\left((1-t)\left|{ }_{a} \mathrm{D}_{q} f(a)\right|+t\left|{ }_{a} \mathrm{D}_{q} f(b)\right|-\mu t(1-t) \eta^{2}(b, a)\right){ }_{0} \mathrm{~d}_{q} t\right] \\
& =\eta(b, a)\left[\left|{ }_{a} \mathrm{D}_{q} f(a)\right|\left(\int_{0}^{\frac{1}{2}}(1-t)\left|q t-\frac{1}{6}\right|{ }_{0} \mathrm{~d}_{q} t+\int_{\frac{1}{2}}^{1}(1-t)\left|q t-\frac{5}{6}\right|{ }_{0} \mathrm{~d}_{q} t\right)\right. \\
& +\left|{ }_{a} \mathrm{D}_{q} f(b)\right|\left(\int_{0}^{\frac{1}{2}} t\left|q t-\frac{1}{6}\right|{ }_{0} \mathrm{~d}_{q} t+\int_{\frac{1}{2}}^{1} t\left|q t-\frac{5}{6}\right|{ }_{0} \mathrm{~d}_{q} t\right) \\
& \left.-\mu \eta^{2}(b, a)\left(\int_{0}^{\frac{1}{2}} t(1-t)\left|q t-\frac{1}{6}\right|{ }_{0} \mathrm{~d}_{q} t+\int_{\frac{1}{2}}^{1} t(1-t)\left|q t-\frac{5}{6}\right|{ }_{0} \mathrm{~d}_{q} t\right)\right] .
\end{aligned}
$$

In view of the Definitions 5 and 7, and Propositions 1 and 2, a direct calculation gives

$$
\begin{gathered}
A_{1}(q)=\int_{0}^{\frac{1}{2}}(1-t)\left|q t-\frac{1}{6}\right|{ }_{0} \mathrm{~d}_{q} t=\left\{\begin{array}{l}
\frac{1-4 q^{3}}{24(1+q)\left(1+q+q^{2}\right)}, 0<q<\frac{1}{3}, \\
\frac{1+12 q+12 q^{2}+36 q^{3}}{216(1+q)\left(1+q+q^{2}\right)}, \frac{1}{3} \leq q<1,
\end{array}\right. \\
A_{2}(q)=\int_{0}^{\frac{1}{2}} t\left|q t-\frac{1}{6}\right|{ }_{0} \mathrm{~d}_{q} t=\left\{\begin{array}{l}
\frac{1-2 q-2 q^{2}}{24(1+q)\left(1+q+q^{2}\right)}, 0<q<\frac{1}{3}, \\
\frac{18 q^{2}+18 q-7}{216(1+q)\left(1+q+q^{2}\right)}, \frac{1}{3} \leq q<1,
\end{array}\right. \\
A_{3}(q)=\int_{0}^{\frac{1}{2}} t(1-t)\left|q t-\frac{1}{6}\right|{ }_{0} \mathrm{~d}_{q} t=\left\{\begin{array}{l}
\frac{1-2 q-2 q^{3}-4 q^{4}}{48(1+q)\left(1+q^{2}\right)\left(1+q+q^{2}\right)}, 0<q<\frac{1}{3}, \\
\frac{108 q^{4}+54 q^{3}+12 q^{2}+54 q-17}{1296(1+q)\left(1+q^{2}\right)\left(1+q+q^{2}\right)}, \frac{1}{3} \leq q<1,
\end{array}\right. \\
A_{4}(q)=\int_{\frac{1}{2}}^{1}(1-t)\left|q t-\frac{5}{6}\right|{ }_{0} \mathrm{~d}_{q} t=\left\{\begin{array}{l}
\frac{-5+8 q+8 q^{2}-8 q^{3}}{24(1+q)\left(1+q+q^{2}\right)}, 0<q<\frac{5}{6}, \\
\frac{12 q^{2}+12 q+5}{216(1+q)\left(1+q+q^{2}\right)}, \frac{5}{6} \leq q<1,
\end{array}\right.
\end{gathered}
$$




$$
\begin{gathered}
A_{5}(q)=\int_{\frac{1}{2}}^{1} t\left|q t-\frac{5}{6}\right|{ }_{0} \mathrm{~d}_{q} t=\left\{\begin{array}{l}
\frac{5-2 q-2 q^{2}}{8(1+q)\left(1+q+q^{2}\right)^{2}}, 0<q<\frac{5}{6}, \\
\frac{18 q^{2}+18 q+25}{216(1+q)\left(1+q+q^{2}\right)}, \frac{5}{6} \leq q<1,
\end{array}\right. \\
A_{6}(q)=\int_{\frac{1}{2}}^{1} t(1-t)\left|q t-\frac{5}{6}\right|{ }_{0} \mathrm{~d}_{q} t= \begin{cases}\frac{5-2 q+28 q^{2}-2 q^{3}-12 q^{4}}{48(1+q)\left(1+q^{2}\right)\left(1+q+q^{2}\right)}, & 0<q<\frac{5}{6}, \\
\frac{108 q^{4}-54 q^{3}+96 q^{2}-54 q+115}{1296(1+q)\left(1+q^{2}\right)\left(1+q+q^{2}\right)}, \frac{5}{6} \leq q<1 .\end{cases}
\end{gathered}
$$

Hence, we deduce the required inequality (5). This completes the proof of Theorem 1.

Theorem 2. Let $f: I=[a, a+\eta(b, a)] \rightarrow \mathbb{R}$ be a q-differentiable function on I with $\eta(b, a)>0$. If $\left|{ }_{a} \mathrm{D}_{q}\right|^{r}$ is an integrable and a generalized strongly preinvex function with modulus $\mu \geq 0, r>1$ and $0<q<1$, then

$$
\begin{aligned}
& \left|\frac{1}{6}\left[f(a)+4 f\left(\frac{2 a+\eta(b, a)}{2}\right)+f(a+\eta(b, a))\right]-\frac{1}{\eta(b, a)} \int_{a}^{a+\eta(b, a)} f(t){ }_{a} \mathrm{~d}_{q} t\right| \\
& \leq \eta(b, a)\left[\left(B_{1}(q)\right)^{1-\frac{1}{r}}\left(A_{1}(q)\left|{ }_{a} \mathrm{D}_{q} f(a)\right|^{r}+A_{2}(q)\left|{ }_{a} \mathrm{D}_{q} f(b)\right|^{r}-\mu A_{3}(q) \eta^{2}(b, a)\right)^{\frac{1}{r}}\right. \\
& \left.\quad+\left(B_{2}(q)\right)^{1-\frac{1}{r}}\left(A_{4}(q)\left|{ }_{a} \mathrm{D}_{q} f(a)\right|^{r}+A_{5}(q)\left|{ }_{a} \mathrm{D}_{q} f(b)\right|^{r}-\mu A_{6}(q) \eta^{2}(b, a)\right)^{\frac{1}{r}}\right]
\end{aligned}
$$

where

$$
\begin{aligned}
& B_{1}(q)=\left\{\begin{array}{l}
\frac{1-2 q}{12(1+q)}, 0<q<\frac{1}{3}, \\
\frac{6 q-1}{36(1+q)}, \frac{1}{3} \leq q<1,
\end{array}\right. \\
& B_{2}(q)=\left\{\begin{array}{l}
\frac{5-4 q}{12(1+q)}, 0<q<\frac{5}{6}, \\
\frac{4 q-5}{12(1+q)}, \frac{5}{6} \leq q<1,
\end{array}\right.
\end{aligned}
$$

$A_{1}(q), A_{2}(q), A_{3}(q), A_{4}(q), A_{5}(q)$, and $A_{6}(q)$ are given by the same expressions as described in Theorem 1.

Proof. Using Lemma 1 and the Hölder inequality, one has

$$
\begin{gathered}
\left|\frac{1}{6}\left[f(a)+4 f\left(\frac{2 a+\eta(b, a)}{2}\right)+f(a+\eta(b, a))\right]-\frac{1}{\eta(b, a)} \int_{a}^{a+\eta(b, a)} f(x){ }_{a} \mathrm{~d}_{q} x\right| \\
=\left|\eta(b, a) \int_{0}^{1} \Psi(t, q)_{a} \mathrm{D}_{q} f(a+t \eta(b, a))_{0} \mathrm{~d}_{q} t\right| \\
=\eta(b, a) \mid \int_{0}^{\frac{1}{2}}\left(q t-\frac{1}{6}\right){ }_{a} \mathrm{D}_{q} f(a+t \eta(b, a))_{0} \mathrm{~d}_{q} t \\
+\int_{\frac{1}{2}}^{1}\left(q t-\frac{5}{6}\right){ }_{a} \mathrm{D}_{q} f(a+t \eta(b, a))_{0} \mathrm{~d}_{q} t \mid
\end{gathered}
$$




$$
\begin{gathered}
\leq \eta(b, a)\left[\left.\int_{0}^{\frac{1}{2}}\left|q t-\frac{1}{6}\right|{ }_{a} \mathrm{D}_{q} f(a+t \eta(b, a))\right|_{0} \mathrm{~d}_{q} t\right. \\
\left.+\left.\int_{\frac{1}{2}}^{1}\left|q t-\frac{5}{6}\right|{ }_{a} \mathrm{D}_{q} f(a+t \eta(b, a))\right|_{0} \mathrm{~d}_{q} t\right] \\
\leq \eta(b, a)\left[\left(\int_{0}^{\frac{1}{2}}\left|q t-\frac{1}{6}\right|{ }_{0} \mathrm{~d}_{q} t\right)^{1-\frac{1}{r}}\left(\left.\int_{0}^{\frac{1}{2}}\left|q t-\frac{1}{6}\right|{ }_{a} \mathrm{D}_{q} f(a+t \eta(b, a))\right|^{r}{ }_{0} \mathrm{~d}_{q} t\right)^{\frac{1}{r}}\right. \\
\left.+\left(\int_{\frac{1}{2}}^{1}\left|q t-\frac{5}{6}\right|{ }_{0} \mathrm{~d}_{q} t\right)^{1-\frac{1}{r}}\left(\left.\int_{\frac{1}{2}}^{1}\left|q t-\frac{5}{6}\right|{ }_{a} \mathrm{D}_{q} f(a+t \eta(b, a))\right|^{r}{ }_{0} \mathrm{~d}_{q} t\right)^{\frac{1}{r}}\right] \\
\times \eta(b, a)\left[\left(\int_{0}^{\frac{1}{2}}\left|q t-\frac{1}{6}\right|{ }_{0} \mathrm{~d}_{q} t\right)^{1-\frac{1}{r}}\right. \\
\times\left(\int_{0}^{\frac{1}{2}}\left|q t-\frac{1}{6}\right|\left[(1-t)\left|{ }_{a} \mathrm{D}_{q} f(a)\right|^{r}+\left.\left.t\right|_{a} \mathrm{D}_{q} f(b)\right|^{r}-\mu t(1-t) \eta^{2}(b, a)\right]{ }_{0} \mathrm{~d}_{q} t\right)^{\frac{1}{r}} \\
+\left(\int_{\frac{1}{2}}^{1}\left|q t-\frac{5}{6}\right|{ }_{0} \mathrm{~d}_{q} t\right)^{1-\frac{1}{r}} \\
\left.\times\left(\int_{\frac{1}{2}}^{1}\left|q t-\frac{5}{6}\right|\left[(1-t)\left|{ }_{a} \mathrm{D}_{q} f(a)\right|^{r}+\left.t{ }_{a} \mathrm{D}_{q} f(b)\right|^{r}-\mu t(1-t) \eta^{2}(b, a)\right]{ }_{0} \mathrm{~d}_{q} t\right)^{\frac{1}{r}}\right]
\end{gathered}
$$

By direct computation, we find

$$
\begin{aligned}
& B_{1}(q)=\int_{0}^{\frac{1}{2}}\left|q t-\frac{1}{6}\right|{ }_{0} \mathrm{~d}_{q} t=\left\{\begin{array}{l}
\frac{1-2 q}{12(1+q)}, 0<q<\frac{1}{3}, \\
\frac{6 q-1}{36(1+q)}, \frac{1}{3} \leq q<1,
\end{array}\right. \\
& B_{2}(q)=\int_{\frac{1}{2}}^{1}\left|q t-\frac{5}{6}\right|{ }_{0} \mathrm{~d}_{q} t=\left\{\begin{array}{l}
\frac{5-4 q}{12(1+q)}, 0<q<\frac{5}{6}, \\
\frac{4 q-5}{12(1+q)}, \frac{5}{6} \leq q<1,
\end{array}\right.
\end{aligned}
$$

and obtain the integral expressions of $A_{1}(q), A_{2}(q), A_{3}(q), A_{4}(q), A_{5}(q)$, and $A_{6}(q)$, which have the same formulas as those given in Theorem 1 . This completes the proof of Theorem 2.

\section{Applications}

It is worth noting that in Definition 4 for $\mu=0$, the generalized strongly preinvex functions reduce to the preinvex functions. Moreover, if we put $\eta=v-u$ in Definition 2, then the preinvex functions reduce to the classical convex functions. Besides, the quantum integral inequalities would lead to the corresponding Riemann integral inequalities by taking the limit $q \rightarrow 1^{-}$. Thus, several new and previously known results can be derived from Theorems 1 and 2 as special cases. Here, we illustrate the applications of our main results by three examples.

Example 1. Recently, Zhang and Du et al. [26] investigated the quantum integral inequalities for convex functions, they established the following inequality: 


$$
\begin{aligned}
& \left|\frac{1}{3}\left[\frac{q f(a)+f(b)}{1+q}+2 f\left(\frac{q a+b}{1+q}\right)\right]-\frac{1}{b-a} \int_{a}^{b} f(t){ }_{a} \mathrm{~d}_{q} t\right| \\
& \leq \min \left\{\mathcal{H}_{1}\left(\frac{1}{3}, \frac{1}{1+q}, 1,1\right), \mathcal{H}_{2}\left(\frac{1}{3}, \frac{1}{1+q}, 1,1\right)\right\},
\end{aligned}
$$

where $f:[a, b] \rightarrow \mathbb{R}$ is a q-differentiable function and $\left|{ }_{a} \mathrm{D}_{q} f\right|$ is an integrable and convex function with $0<q<1$, the expressions of $\mathcal{H}_{1}$ and $\mathcal{H}_{2}$ are given by [26] (Theorem 3.2).

Further, in [26], the authors derived a remarkable inequality from (7), as follows:

$$
\left|\frac{1}{3}\left[\frac{f(a)+f(b)}{2}+2 f\left(\frac{a+b}{2}\right)\right]-\frac{1}{b-a} \int_{a}^{b} f(t) d t\right| \leq \frac{5(b-a)}{72}\left[\left|f^{\prime}(a)\right|+\left|f^{\prime}(b)\right|\right]
$$

where $f:[a, b] \rightarrow \mathbb{R}$ is a differentiable function, and $\left|f^{\prime}\right|$ is an integrable and convex function on $[a, b]$.

In the following, we show a new result analogous to the inequality (7), which can be obtained directly by taking $\mu=0$ in Theorem 1 .

Corollary 1. Let $f: I=[a, a+\eta(b, a)] \rightarrow \mathbb{R}$ be a q-differentiable function on I with $\eta(b, a)>0$. If $\left|{ }_{a} \mathrm{D}_{q} f\right|$ is an integrable and preinvex function, $0<q<1$, then

$$
\begin{aligned}
& \left|\frac{1}{3}\left[\frac{f(a)+f(a+\eta(b, a))}{2}+2 f\left(\frac{2 a+\eta(b, a)}{2}\right)\right]-\frac{1}{\eta(b, a)} \int_{a}^{a+\eta(b, a)} f(t){ }_{a} \mathrm{~d}_{q} t\right| \\
& \leq \eta(b, a)\left[\left(A_{1}(q)+A_{4}(q)\right)\left|{ }_{a} \mathrm{D}_{q} f(a)\right|+\left(A_{2}(q)+A_{5}(q)\right)\left|{ }_{a} \mathrm{D}_{q} f(b)\right|\right]
\end{aligned}
$$

where $A_{1}(q), A_{2}(q), A_{4}(q)$, and $A_{5}(q)$ are the coefficients as described in Theorem 1.

Putting $\eta(b, a)=b-a$ in Corollary 1 , it follows that

Corollary 2. Let $f:[a, b] \rightarrow \mathbb{R}$ be a q-differentiable function. If $\left|{ }_{a} \mathrm{D}_{q} f\right|$ is an integrable and convex function, $0<q<1$, then

$$
\begin{aligned}
& \left|\frac{1}{3}\left[\frac{f(a)+f(b)}{2}+2 f\left(\frac{a+b}{2}\right)\right]-\frac{1}{b-a} \int_{a}^{b} f(t){ }_{a} \mathrm{~d}_{q} t\right| \\
& \leq(b-a)\left[\left(A_{1}(q)+A_{4}(q)\right)\left|{ }_{a} \mathrm{D}_{q} f(a)\right|+\left(A_{2}(q)+A_{5}(q)\right)\left|{ }_{a} \mathrm{D}_{q} f(b)\right|\right],
\end{aligned}
$$

where $A_{1}(q), A_{2}(q), A_{4}(q)$, and $A_{5}(q)$ are the coefficients as described in Theorem 1.

Remark 1. In Corollary 2, if we take the limit $q \rightarrow 1^{-}$in (10) and use the basic properties of $q$-derivative and q-integral ([25], see also [1])

$$
\lim _{q \rightarrow 1^{-}}{ }_{a} D_{q} f(t)=f^{\prime}(t), \quad \lim _{q \rightarrow 1^{-}} \int_{a}^{b} f(t){ }_{a} \mathrm{~d}_{q} t=\int_{a}^{b} f(t) d t
$$

along with the equalities

$$
\lim _{q \rightarrow 1^{-}}\left(A_{1}(q)+A_{4}(q)\right)=\lim _{q \rightarrow 1^{-}}\left(\frac{1+12 q+12 q^{2}+36 q^{3}}{216(1+q)\left(1+q+q^{2}\right)}+\frac{12 q^{2}+12 q+5}{216(1+q)\left(1+q+q^{2}\right)}\right)=\frac{5}{72}
$$




$$
\lim _{q \rightarrow 1^{-}}\left(A_{2}(q)+A_{5}(q)\right)=\lim _{q \rightarrow 1^{-}}\left(\frac{18 q^{2}+18 q-7}{216(1+q)\left(1+q+q^{2}\right)}+\frac{18 q^{2}+18 q+25}{216(1+q)\left(1+q+q^{2}\right)}\right)=\frac{5}{72}
$$

then we obtain the inequality

$$
\left|\frac{1}{3}\left[\frac{f(a)+f(b)}{2}+2 f\left(\frac{a+b}{2}\right)\right]-\frac{1}{b-a} \int_{a}^{b} f(t) d t\right| \leq \frac{5(b-a)}{72}\left[\left|f^{\prime}(a)\right|+\left|f^{\prime}(b)\right|\right] .
$$

This is exactly the above-mentioned inequality (8) due to Zhang and Du et al. [26].

Example 2. In a recent paper [27], Tunç, Göv, and Balgeçti established a Simpson-type quantum integral inequality for convex functions ([27] Theorem 1), as follows:

$$
\begin{gathered}
\left|\frac{1}{6}\left[f(a)+4 f\left(\frac{a+b}{2}\right)+f(b)\right]-\frac{1}{b-a} \int_{a}^{b} f(t){ }_{a} \mathrm{~d}_{q} t\right| \\
\leq \frac{(b-a)}{12}\left[\frac{2 q^{2}+2 q+1}{q^{3}+2 q^{2}+2 q+1}\left|{ }_{a} \mathrm{D}_{q} f(b)\right|+\left.\frac{1}{3} \cdot \frac{6 q^{3}+4 q^{2}+4 q+1}{q^{3}+2 q^{2}+2 q+1}\right|_{a} \mathrm{D}_{q} f(a) \mid\right],
\end{gathered}
$$

where $f:[a, b] \rightarrow \mathbb{R}$ is a continuous function, $\left|{ }_{a} \mathrm{D}_{q} f\right|$ is a convex and integrable function with $0<q<1$.

Remark 2. Before we describe the related result of inequality (12), we should point out that in (12) there is an error occurring in the coefficients of $\left|{ }_{a} \mathrm{D}_{q} f(b)\right|$ and $\left|{ }_{a} \mathrm{D}_{q} f(a)\right|$. The mistakes arise from the calculations of quantum integrals in [27] (Lemmas 4 and 5), the details are as follows:

As an auxiliary for establishing the inequality (12), in [27] (Lemmas 4 and 5), the authors gave the following results involving $q$-integrals $(0<q<1)$ :

$$
\begin{aligned}
\int_{0}^{\frac{1}{2}}(1-t)\left|q t-\frac{1}{6}\right|{ }_{0} \mathrm{~d}_{q} t & =\frac{36 q^{3}+12 q^{2}+12 q+1}{216\left(q^{3}+2 q^{2}+2 q+1\right)}, \\
\int_{\frac{1}{2}}^{1}(1-t)\left|q t-\frac{5}{6}\right|{ }_{0} \mathrm{~d}_{q} t & =\frac{12 q^{2}+12 q+5}{216\left(q^{3}+2 q^{2}+2 q+1\right)} .
\end{aligned}
$$

However, the equality (13) is incorrect for the case of $0<q<\frac{1}{3}$; and the equality (14) is incorrect for the case of $0<q<\frac{5}{6}$, which can be observed by direct computation of $q$-integrals. In fact, by the formulas and algorithms for $q$-integrals stated in Propositions 1 and 2 , when $0<q<\frac{1}{3}$, we have

$$
\begin{aligned}
& \int_{0}^{\frac{1}{2}}(1-t)\left|q t-\frac{1}{6}\right|{ }_{0} \mathrm{~d}_{q} t \\
= & \int_{0}^{\frac{1}{2}}(1-t)\left(\frac{1}{6}-q t\right){ }_{0} \mathrm{~d}_{q} t \\
= & \int_{0}^{\frac{1}{2}}\left(q t^{2}-\frac{1}{6} t-q t+\frac{1}{6}\right){ }_{0} \mathrm{~d}_{q} t \\
= & q \int_{0}^{\frac{1}{2}} t^{2}{ }_{0} \mathrm{~d}_{q} t-\left(\frac{1}{6}+q\right) \int_{0}^{\frac{1}{2}} t_{0} \mathrm{~d}_{q} t+\frac{1}{6} \int_{0}^{\frac{1}{2}} 1_{0} \mathrm{~d}_{q} t \\
= & q \frac{1}{8\left(q+q^{2}+1\right)}-\left(\frac{1}{6}+q\right) \frac{1}{4(1+q)}+\frac{1}{12} \\
= & \frac{1-4 q^{3}}{24\left(q^{3}+2 q^{2}+2 q+1\right)} .
\end{aligned}
$$


When $0<q<\frac{5}{6}$, we have

$$
\begin{aligned}
& \int_{\frac{1}{2}}^{1}(1-t)\left|q t-\frac{5}{6}\right|{ }_{0} \mathrm{~d}_{q} t \\
= & \int_{\frac{1}{2}}^{1}(1-t)\left(\frac{5}{6}-q t\right){ }_{0} \mathrm{~d}_{q} t \\
= & \int_{\frac{1}{2}}^{1}\left(q t^{2}-\frac{1}{6} t-q t+\frac{1}{6}\right){ }_{0} \mathrm{~d}_{q} t \\
= & q \int_{\frac{1}{2}}^{1} t^{2}{ }_{0} \mathrm{~d}_{q} t-\left(\frac{5}{6}+q\right) \int_{\frac{1}{2}}^{1} t_{0} \mathrm{~d}_{q} t+\frac{5}{6} \int_{\frac{1}{2}}^{1} 1_{0} \mathrm{~d}_{q} t \\
= & q\left(\int_{0}^{1} t^{2}{ }_{0} \mathrm{~d}_{q} t-\int_{0}^{\frac{1}{2}} t^{2}{ }_{0} \mathrm{~d}_{q} t\right)-\left(\frac{5}{6}+q\right)\left(\int_{0}^{1} t_{0} \mathrm{~d}_{q} t-\int_{0}^{\frac{1}{2}} t_{0} \mathrm{~d}_{q} t\right)+\frac{5}{12} \\
= & q\left(\frac{1}{q+q^{2}+1}-\frac{1}{8\left(q+q^{2}+1\right)}\right)-\left(\frac{5}{6}+q\right)\left(\frac{1}{1+q}-\frac{1}{4(1+q)}\right)+\frac{5}{12} \\
= & \frac{-5+8 q+8 q^{2}-8 q^{3}}{24\left(q^{3}+2 q^{2}+2 q+1\right)} .
\end{aligned}
$$

In the same way, one can verify that the equality (13) is valid for $\frac{1}{3} \leq q<1$, the equality (14) is valid for $\frac{5}{6} \leq q<1$.

In the following we provide a modified version of inequality (12).

Corollary 3. Let $f:[a, b] \rightarrow \mathbb{R}$ be a $q$-differentiable function. If $\left|{ }_{a} \mathrm{D}_{q} f\right|$ is an integrable and convex function, $0<q<1$, then

$$
\begin{aligned}
& \left|\frac{1}{3}\left[\frac{f(a)+f(b)}{2}+2 f\left(\frac{a+b}{2}\right)\right]-\frac{1}{b-a} \int_{a}^{b} f(t){ }_{a} \mathrm{~d}_{q} t\right| \\
& \leq(b-a)\left[C_{1}(q)\left|{ }_{a} \mathrm{D}_{q} f(a)\right|+C_{2}(q)\left|{ }_{a} \mathrm{D}_{q} f(b)\right|\right],
\end{aligned}
$$

where $C_{1}(q)$ and $C_{2}(q)$ are given by

$$
\begin{gathered}
C_{1}(q)= \begin{cases}\frac{-3 q^{3}+2 q^{2}+2 q-1}{6\left(q^{3}+2 q^{2}+2 q+1\right)}, & 0<q<\frac{1}{3}, \\
\frac{-9 q^{3}+21 q^{2}+21 q-11}{54\left(q^{3}+2 q^{2}+2 q+1\right)}, & \frac{1}{3} \leq q<\frac{5}{6} \\
\frac{6 q^{3}+4 q^{2}+4 q+1}{36\left(q^{3}+2 q^{2}+2 q+1\right)}, & \frac{5}{6} \leq q<1 .\end{cases} \\
C_{2}(q)= \begin{cases}\frac{-q^{2}-q+2}{3\left(q^{3}+2 q^{2}+2 q+1\right)}, & 0<q<\frac{1}{3}, \\
\frac{-9 q^{2}-9 q+32}{54\left(q^{3}+2 q^{2}+2 q+1\right)}, & \frac{1}{3} \leq q<\frac{5}{6} \\
\frac{2 q^{2}+2 q+1}{12\left(q^{3}+2 q^{2}+2 q+1\right)}, & \frac{5}{6} \leq q<1 .\end{cases}
\end{gathered}
$$

Proof. Using Corollary 2 and performing a simple calculation in the expressions $C_{1}(q)=A_{1}(q)+$ $A_{4}(q)$ and $C_{2}(q)=A_{2}(q)+A_{5}(q)$, where $A_{1}(q), A_{2}(q), A_{4}(q)$, and $A_{5}(q)$ are the coefficients from Theorem 1 , we obtain the inequality (15).

Example 3. We provide an estimation of upper bound for the q-integral $\int_{a}^{a+\eta(b, a)} f(t){ }_{a} \mathrm{~d}_{q} t$.

Corollary 4. Let $f: I=[a, a+\eta(b, a)] \rightarrow \mathbb{R}$ be a q-differentiable function on I with $\eta(b, a)>0$. If $\left|{ }_{a} \mathrm{D}_{q} f\right|$ is an integrable and generalized strongly preinvex function with modulus $\mu \geq 0$ and $0<q<1$, then 


$$
\begin{aligned}
& \left|\int_{a}^{a+\eta(b, a)} f(t){ }_{a} \mathrm{~d}_{q} t\right| \leq \eta(b, a)\left|\frac{1}{6}\left[f(a)+4 f\left(\frac{2 a+\eta(b, a)}{2}\right)+f(a+\eta(b, a))\right]\right| \\
& +\eta^{2}(b, a)\left[\left(A_{1}(q)+A_{4}(q)\right)\left|{ }_{a} \mathrm{D}_{q} f(a)\right|+\left(A_{2}(q)+A_{5}(q)\right)\left|{ }_{a} \mathrm{D}_{q} f(b)\right|-\mu\left(A_{3}(q)+A_{6}(q)\right) \eta^{2}(b, a)\right],
\end{aligned}
$$

where $A_{1}(q), A_{2}(q), A_{3}(q), A_{4}(q), A_{5}(q)$ and $A_{6}(q)$ are the coefficients as described in Theorem 1.

Proof. Note that

$$
\begin{aligned}
& \left|\frac{1}{\eta(b, a)} \int_{a}^{a+\eta(b, a)} f(t){ }_{a} \mathrm{~d}_{q} t\right| \leq\left|\frac{1}{6}\left[f(a)+4 f\left(\frac{2 a+\eta(b, a)}{2}\right)+f(a+\eta(b, a))\right]\right| \\
& +\left|\frac{1}{\eta(b, a)} \int_{a}^{a+\eta(b, a)} f(t){ }_{a} \mathrm{~d}_{q} t-\frac{1}{6}\left[f(a)+4 f\left(\frac{2 a+\eta(b, a)}{2}\right)+f(a+\eta(b, a))\right]\right| .
\end{aligned}
$$

Utilizing Theorem 1 , one has

$$
\begin{gathered}
\left|\frac{1}{\eta(b, a)} \int_{a}^{a+\eta(b, a)} f(t)_{a} \mathrm{~d}_{q} t\right| \leq\left|\frac{1}{6}\left[f(a)+4 f\left(\frac{2 a+\eta(b, a)}{2}\right)+f(a+\eta(b, a))\right]\right| \\
+\eta(b, a)\left[\left.\left(A_{1}(q)+A_{4}(q)\right)\right|_{a} \mathrm{D}_{q} f(a)\left|+\left(A_{2}(q)+A_{5}(q)\right)\right|{ }_{a} \mathrm{D}_{q} f(b) \mid-\mu\left(A_{3}(q)+A_{6}(q)\right) \eta^{2}(b, a)\right] .
\end{gathered}
$$

Multiplying both sides of the above inequality by $\eta(b, a)$ leads to the desired inequality (16).

Author Contributions: Y.D., M.U.A., and S.W. finished the proofs of the main results and the writing work. Both authors contributed equally to the writing of this paper. All authors read and approved the final manuscript.

Funding: This work was supported by the Teaching Reform Project of Longyan University (Grant No. 2017JZ02) and the Teaching Reform Project of Fujian Provincial Education Department (Grant No. FBJG20180120).

Acknowledgments: The authors would like to express sincere appreciation to the editors and the anonymous reviewers for their valuable comments and suggestions which helped to improve the manuscript.

Conflicts of Interest: The authors declare no conflict of interest.

\section{References}

1. Kac, V.; Cheung, P. Quantum Calculus; Springer: New York, NY, USA, 2002.

2. Ernst, T. A Comprehensive Treatment of $q$-Calculus; Birkhäuser: Basel, Switzerland; New York, NY, USA, 2012.

3. Ernst, T. A method for $q$-calculus. J. Nonlinear Math. Phys. 2003, 10, 487-525. [CrossRef]

4. Alp, N.; Sarikaya, M.Z.; Kunt, M.; Iscan, I. q-Hermite Hadamard inequalities and quantum estimates for midpoint type inequalities via convex and quasi-convex functions. J. King Saud Univ. Sci. 2018, 30, 193-203. [CrossRef]

5. Lee, J.; Vîlcu, G.E. Inequalities for generalized normalized $\delta$-Casorati curvatures of slant submanifolds in quaternionic space forms. Taiwan J. Math. 2015, 19, 691-702. [CrossRef]

6. Vîlcu, G.E. An optimal inequality for Lagrangian submanifolds in complex space forms involving Casorati curvature. J. Math. Anal. Appl. 2018, 465, 1209-1222. [CrossRef]

7. Aquib, M.; Lee, J.E.; Vîlcu, G.E.; Yoon, D.W. Classification of Casorati ideal Lagrangian submanifolds in complex space forms. Differ. Geom. Appl. 2019, 63, 30-49. [CrossRef]

8. Vîlcu, A.D.; Vîlcu, G.E. On quasi-homogeneous production functions. Symmetry 2019, 11, 976. [CrossRef]

9. Hanson, M.A. On sufficiency of the Kuhn-Tucker conditions. J. Math. Anal. Appl. 1981, 80, 545-550. [CrossRef]

10. Jeyakumar, V. Strong and weak invexity in mathematical programming. Methods Oper. Res. 1985, 55, 109-125.

11. Ben-Israel, A.; Mond, B. What is invexity? J. Austral. Math. Soc. Ser. B 1986, 28, 1-9. [CrossRef]

12. Weir, T.; Mond, B. Preinvex functions in multiple objective optimization. J. Math. Anal. Appl. 1988, 136, 29-38. [CrossRef] 
13. Jeyakumar, V.; Mond, B. On generalized convex mathematical programming. J. Aust. Math. Soc. Ser. B 1992, 34, 43-53. [CrossRef]

14. Noor, M.A. Generalized convex functions. Pan-Am. Math. J. 1994, 4, 73-89.

15. Mohan, S.R.; Neogy, S.K. On invex sets and preinvex functions. J. Math. Anal. Appl. 1995, 189, 901-908. [CrossRef]

16. Noor, M.A.; Noor, K.I. Some characterizations of strongly preinvex functions. J. Math. Anal. Appl. 2006, 316, 697-706. [CrossRef]

17. Awan, M.U.; Cristescu, G.; Noor, M.A.; Riahi, L. Upper and lower bounds for Riemann type quantum integrals of preinvex and preinvex dominated functions. UPB Sci. Bull. Ser. A 2017, 79, 33-44.

18. Cristescu, G.; Noor, M.A.; Awan, M.U. Bounds of the second degree cumulative frontier gaps of functions with generalized convexity. Carpath. J. Math. 2015, 31, 173-180.

19. Niculescu, C.P.; Persson, L.E. Convex Functions and Their Applications, A Contemporary Approach, 2nd ed.; CMS Books in Mathematics; Springer: New York, NY, USA, 2018; Volume 23.

20. Cristescu, G.; Lupsa, L. Non-Connected Convexities and Applications; Kluwer Academic Publishers: Dordrecht, The Netherlands, 2002.

21. Noor, M.A.; Noor, K.I.; Awan, M.U. Some quantum integral inequalities via preinvex functions. Appl. Math.Comput. 2015, 269, 242-251. [CrossRef]

22. Noor, M.A.; Noor, K.I.; Awan, M.U. Quantum Ostrowski inequalities for $q$-differentiable convex functions. J. Math. Inequal. 2016, 10, 1013-1018. [CrossRef]

23. Riahi, L.; Awan, M.U.; Noor, M.A. Some complementary $q$-bounds via different classes of convex functions. UPB Sci. Bull. Ser. A 2017, 79, 171-182.

24. Sarikaya, M.Z.; Set, E.; Ozdemir, M.E. On new inequalities of Simpson's type for s-convex functions. Comput. Math. Appl. 2010, 60, 2191-2199. [CrossRef]

25. Tariboon, J.; Ntouyas, S.K. Quantum integral inequalities on finite intervals. J. Inequal. Appl. 2014, $2014,121$. [CrossRef]

26. Zhang, Y.; Du, T.S.; Wang, H.; Shen, Y.J. Different types of quantum integral inequalities via $\alpha, m$-convexity. J. Inequal. Appl. 2018, 2018, 264. [CrossRef] [PubMed]

27. Tunç, M.; Göv, E.; Balgeçti, S. Simpson type quantum integral inequalities for convex functions. Miskolc Math. Notes 2018, 19, 649-664. [CrossRef]

(C) 2019 by the authors. Licensee MDPI, Basel, Switzerland. This article is an open access article distributed under the terms and conditions of the Creative Commons Attribution (CC BY) license (http:/ / creativecommons.org/licenses/by/4.0/). 